\title{
Backward masking: The role of the target + mask composite
}

\author{
STEPHEN HANDEL \\ The University of Tennessee, Knoxville, Tennessee 37916
}

\begin{abstract}
In a backward masking reproduction task, the target was a 4-dot pattern placed in a $3 \times$ array and the mask was a single dot placed in one of the five remaining empty cells of that array. The target + mask composite is thereby a 5-dot pattern. The results demonstrated that redundant 4-dot patterns were easier to reproduce. Moreover, the redundancy of the composite affects accuracy. Reproduction was most accurate when the redundancy of the target matched the redundancy of the composite. Thus, the structural characteristics of the whole determined the perception and realization of the subparts.
\end{abstract}

The central theme of Gestalt psychology is that of form. Form is the way things appear; they have a "whole-character" which transcends the characteristics of the parts, and the perception of form is not learning to put the whole together out of pieces. The most widely used demonstrations that experience is at best a secondary influence on form perception are Gottschaldt figures (1926). A simple, very familiar figure, when skillfully embedded in other lines, is often not perceived. The simple figure is not seen since "field forces" produce a new whole, effectively camouflaging the original figure.

The visual masking paradigm can be considered an experimental procedure producing the Gottschaldt demonstrations. An overlearned (target) stimulus is presented, followed in close temporal proximity (within $200 \mathrm{msec}$ ) by a masking stimulus creating a composite of the two stimuli. The subject's task is to identify the target stimulus. The similarity of these two lines of research suggests that it is possible to study form perception utilizing backward masking. Specifically, it is possible to study the characteristics of the target + mask composite which precludes the identification of the target, much in the same fashion as the Gottschaldt demonstrations.

The stimuli used here were 4-dot and 5-dot patterns generated by placing four or five dots in the cells of a 3 x 3 matrix. A total of 1264 -dot and 5-dot patterns are possible. These patterns can be partitioned into equivalence sets by a physical criteria based on rotation and reflection (Prokhovnik, 1959). Two patterns are equivalent if one can be transformed into the other by one or more $90-\mathrm{deg}$ rotations and or reflections around the vertical or horizontal axes. Using these criteria, the 126 patterns may be partitioned into 23 mutually

This research was supported by a National Institute of Health Biomedical Support Grant to The University of Tennessee. The author would like to thank John Malone for his helpful comments in preparing the manuscript. Requests for reprints should be sent to Stephen Handel, Department of Psychology, The University of Tennessee, Knoxville, Tennessee 37916. exclusive equivalence sets. Each set contains one, four, or eight different patterns which are equivalent by the rotation/reflection criteria.

Garner (1962) originally suggested that the goodness of any pattern was inversely related to the number of psychologically equivalent patterns. The smaller the equivalence set, the greater the structure or redundancy of these patterns relative to the total set of stimuli. Subsequent work has confirmed this hypothesis as: (a) patterns from the same rotation/reflection set were judged equally good; (b) patterns from sets of one stimulus were judged best, patterns from sets of four stimuli intermediate and patterns from sets of eight stimuli were judged poorest (Handel \& Garner, 1966); (c) good patterns are easier to sort into categories (Clement \& Varnodoe, 1967), produce faster recognition time (Checkosky \& Whitlock, 1973), and were easier to reproduce in a backward masking task (Bell \& Handel, Reference Note 1).

The procedure used in the present experiment was to present a 4-dot pattern as the target stimulus and use a single dot in one of the five remaining blank cells of the $3 \times 3$ matrix as the masking stimulus. The target + mask stimulus is therefore a 5-dot pattern. The subject's task was to identify the original 4-dot pattern. The possibilities are illustrated in Figure 1. The 4-dot patterns are shown on the left with the size of the equivalence set and pattern goodness indicated. The five possible 5-dot target + mask composites are shown on the right with the size of equivalence set and pattern goodness also illustrated. It is important to note that any given 4-dot pattern, when combined with a fifth dot, can produce 5-dot patterns from equivalence sets of differing size and therefore of differing redundancy and structure. The purpose of the present study is to investigate the relation between the size of the equivalence set of the target stimulus (i.e., the goodness of the target) and the size of the 5-dot target + mask composite (the goodness of the composite) in which the target stimulus is embedded on pattern identification. 


\section{METHOD}

\section{Subjects}

The eight subjects were University of Tennessee undergraduates. All subjects had normal vision.

\section{Target patterns}

The 4-dot target patterns are shown in Figure 1. For equivalence sets with one or four patterns, all patterns were used. For patterns with eight equivalents, four of the patterns were used.

The target and mask were presented binocularly in an $800 \mathrm{~F}$ two-field Scientific Prototype tachistoscope as white dots against a black background. The center of each cell of the imagery $3 \times 3$ matrix was separated by $2 \mathrm{deg}$ with each dot subtending $.54 \mathrm{deg}$. The masking dot was identical and was placed in the center of the cell. The luminance of the white dots and black surround were $.71 \mathrm{fL}$ and $.04 \mathrm{fL}$, respectively. The target and mask stimuli were presented for $15 \mathrm{msec}$ with an interstimulus interval of 25 msec. A faint red light serving as a fixation point, centered at the

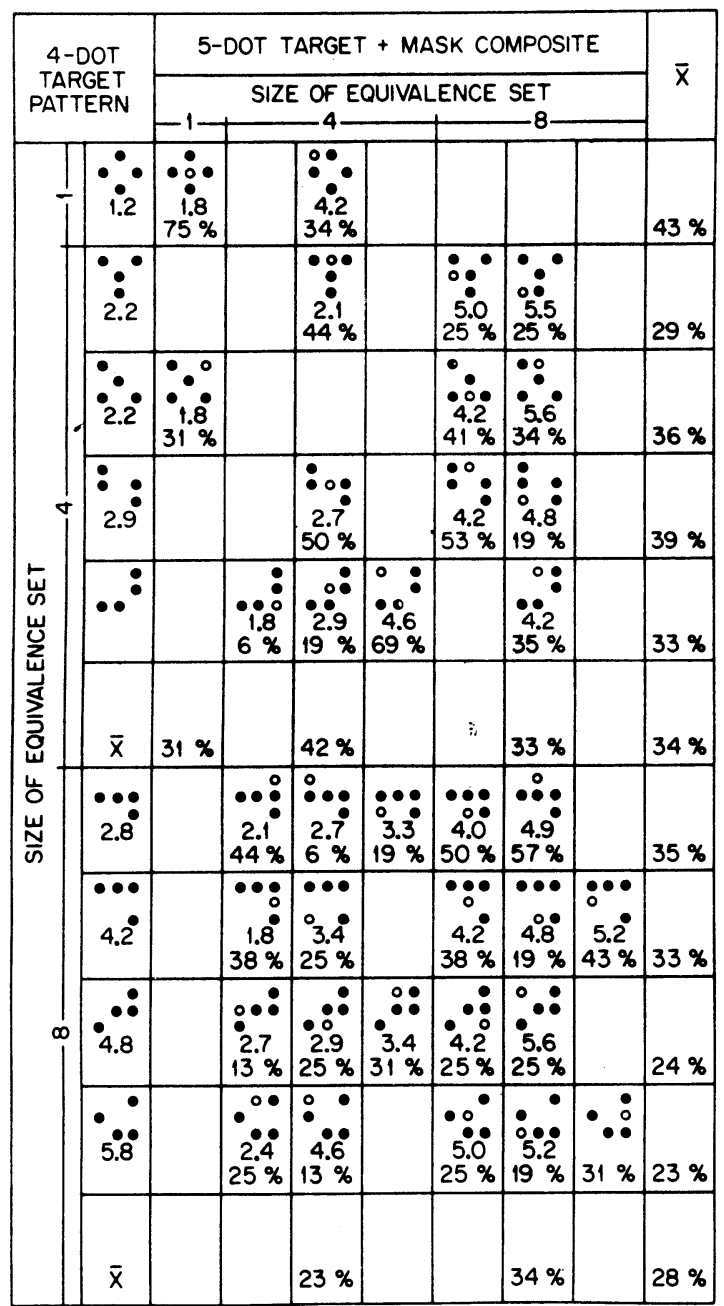

Figure 1. Percentage correct reproduction for each target pattern and possible target + mask composite. The target pattern is represented by filled-in dots and the masking dot is represented by an open dot. The goodness ratings (from Bell and Handel, Reference Note 1) or each target and target + mask composite are presented directly below each pattern. top of the pattern matrix, terminated at the onset of the presentation of the target stimulus.

\section{Procedure}

There was a total of 165 stimulus conditions: 33 patterns $x 5$ masking dots. Each subject received 85 presentations composed of one-half the conditions for equivalence sets of four or eight patterns (80 in all) and the five conditions for the equivalence set with one unique pattern. The orders of presentation were randomized with the restriction that the two instances of each pattern be separated by at least five different patterns and were counterbalanced across subjects.

Subjects were run individually for three sessions and instructed to reproduce as accurately as possible the first pattern of four dots even though it would be followed by a fifth dot. Subjects checked the appropriate cells in a blank $3 \times 3$ matrix drawn on their answer sheets.

In addition, prior to the experimental conditions, two patterns from each equivalence set were presented for $15 \mathrm{msec}$ without being followed by a mask. Subjects attempted to reproduce the patterns on a blank $3 \times 3$ matrix. The results indicated essentially perfect identification of the 4-dot patterns.

\section{RESULTS}

The measure of performance was the percentage of patterns correctly reproduced. Preliminary analysis indicated that the appropriate level of data analyses, in terms of the percentage of correct reproductions, was the size of the target pattern equivalence set and the size of the target + mask composite equivalence set.

\section{Target patterns}

There were significant differences in the percentage of correct reproductions among target patterns from equivalence sets of different size, $[F(2 / 14)=6.2$, $\mathrm{p}<.02]$. Patterns with fewer equivalents were more accurately reproduced. Individual comparisons indicated that all three differences among equivalence sets were significant at the .05 level or better (a priori t tests).

\section{Target + mask composite}

There was an interaction between the size of the equivalence set of the 4-dot pattern and the 5-dot target + mask composite. In short, reproduction was most accurate when the equivalence sets of the target and composite were the same size. For targets with one equivalent, composites with one equivalent produced better reproduction than composites with four equivalents $(75 \%$ to $34 \%, \mathrm{z}=2.1, \mathrm{p}<.05)$; for targets with four equivalents, composites with four equivalents produced better performance than composites with one or eight equivalents (42\% to $31 \%$ or $33 \%$ ) although the differences were not significant; for targets with eight equivalents, composites with eight equivalents produced better performance than composites with four equivalents $[34 \%$ to $23 \%, \mathrm{t} \quad(7)=2.25, \mathrm{p}<.05]$. Moreover, six of the eight subjects produced the major diagonal profile, i.e. the best reproduction occurred when the 5-dot composite had the identical number of equivalents as did the 4-dot target. 


\section{Reproduction errors}

There were no consistent types of errors. The percentage correct did not vary as a function of the position of the masking dot or whether the masking dot completed two lines, one line, or was unconnected to the target elements. Moreover, an incorrectly reported 4-dot pattern did not consistently come from an equivalence set of smaller size, so that the reported patterns were not patterns of higher goodness.

\section{DISCUSSION}

The present results further confirm that good patterns, being more redundant and highly structured, are easier to reproduce than poor patterns under presentation conditions which degrade or interrupt the stimulus. Without masking, there were no differences in reproduction among the target patterns indicating no inherent differences in discriminability. These results replicate in all aspects those of Bell and Handel (Reference Note 1).

In addition, the optimal reproduction occurred when the target + mask composite was from the same size equivalence set as the target. That is, the target and target + mask were of equal goodness, redundancy, or degree of organization. Given this fit, subjects were best able to perceive the target in the composite. In Gottschald t's research, a stimulus composite which had strong internal unity optimally comouflaged the target. The present work gives a slightly different view. A composite which had a different amount of organization produced the most interference; it did not matter whether the composite had more of less structure.

Alternately, the relationship between the target and target + mask composite can be studied by assessing the "fit" between target and composite. Given a 4-dot pattern, what fifth element is implied by the four elements? Or, given a 5-dot pattern, which element should be removed to produce a 4-dot pattern? In the former case, it could be hypothesized that if the fifth masking dot was not implied by the 4-dot pattern, then it would be easier to identify the target as the masking dot might become a "ground element" and not be integrated with the 4-dot target. Bear (1973) has studied the implication task and has found that for good patterns, the implied dot elements were more predictable than for poor patterns. Moreover, the 5-dot pattern tended to be of higher goodness than the 4-dot pattern.
However, there was no correlation between the percentage of times a fifth dot was implied by a 4-dot pattern (from Bear's data) and the probability of correctly identifying the 4-dot pattern when masked by that dot (from present experiment and shown in Figure 1).

It appears that implication and reproduction tasks tap different processes: in an implication task, subjects respond with a dot (or a pattern, Handel \& Garner, 1966) such that the produced pattern is better structured; implication tasks tap the processes leading to greater redundancy and simplicity. In a reproduction task, subjects respond at the same level of structure or redundancy; reproduction tasks tap the processes underlying stimulis similarity and equivalence. It is probably for this reason that reproduction experiments attempting to study the autochronous forces in memory producing better figures have been largely unsuccessful (reviewed by Riley, 1951). By utilizing a reproduction task, these studies have disallowed those factors leading to greater structure and simplicity to operate.

\section{REFERENCE NOTE}

Bell, H. H., \& Handel, S. The role of pattern goodness in the reproduction of backward masked patterns. Manuscript submitted for publication, 1975 .

\section{REFERENCES}

Bear, G. Figural goodness and the predictability of figural elements. Perception \& Psy chophysics, 1973, 13, 32-40.

Checkosky, S. F., \& Whitlock, D. The effects of pattern goodness on recognition time in a memory search task. Journal of Experimental Psy chology, 1973, 100, 341-348.

Clement, D. E., \& Varnadoe, K. W. Pattern uncertainty and the discrimination of visual patterns. Perception \& Psychophysics, 1967, 2, 427-431.

Garner, W. R. Uncertainty and structure as psychological concepts. New York: Wiley, 1962.

Gottschaldt, $K$. Uber den einfluss der Erfahrung auf die Wahrnehmung von Figuren I. Psychologische Forschung, 1926, 8, 261-317. Translated in W. D. Ellis, $A$ source book of Gestalt psychology. 1955

Handel, S., \& Garner, W. R. The structure of visual pattern associates and pattern goodness. Perception \& Psychophysics, $1966,1,33-38$.

Prokhovnik, S. J. Pattern variants on a square field. Psy chom etrika, 1959, 24, 329-341.

Riley, D. A. Memory for form. In C. Postman (Ed.), Psychology in the making. New York: Knopf, 1962.

(Received for publication February 18, 1975.) 